\title{
Biomimetics: extending nature's design of thin-wall shells with cellular cores
}

\author{
M. A. Dawson ${ }^{1} \&$ L. J. Gibson ${ }^{2}$ \\ ${ }^{1}$ Department of Mechanical Engineering, \\ Massachusetts Institute of Technology, USA \\ ${ }^{2}$ Department of Materials Science and Engineering, \\ Massachusetts Institute of Technology, USA
}

\begin{abstract}
Thin-walled, cylindrical structures are found extensively in both engineering and nature. Minimum weight design of such structures is essential in a variety of engineering applications, including space shuttle fuel tanks, aircraft fuselages, and offshore oil platforms. In nature, thin-walled cylindrical structures are often supported by a honeycomb- or foam-like cellular core, as for example, in plant stems, porcupine quills, or hedgehog spines. Previous studies have suggested that a compliant core increases the elastic buckling resistance of a cylindrical shell over that of a hollow cylinder of the same weight. We extend the linearelastic buckling theory by coupling basic plasticity theory to provide a more comprehensive analysis of isotropic, cylindrical shells with compliant cores. The minimum weight design of a thin-walled cylinder with a compliant core, of given radius and specified materials, subjected to a prescribed load in uniaxial compression or pure bending is examined. The analysis gives the values of the shell thickness, the core thickness, and the core density that minimize the weight of the structure for both loading scenarios. The weight optimization of the structure identifies the optimum ratio of the core modulus to the shell modulus. The design of natural, thin-walled structures with cellular cores is compared to the analytical optimal, and the deviation about the theoretical optimum is explored. The analysis also discusses the selection of materials in the design of the cylinders with compliant cores, identifying the most suitable material combinations. Finally, the challenges associated with achieving the optimal design in practice are discussed, and the potential for practical implementation is explored.
\end{abstract}

Keywords: biomimetic, buckling, cellular, core, compliant, cylinder, shell, thin, wall. 


\section{Nomenclature}

a Radius to mid-plane of thickness shell

E Young's Modulus of the shell axial load

$\mathrm{E}_{\mathrm{c}}$ Young's modulus of the core

$\mathrm{E}_{\mathrm{s}}$ Young's modulus of the solid core

$\mathrm{M}_{\mathrm{lb}}$ Buckling moment of shell \& core

$\mathrm{M}_{\mathrm{eq}}$ Buckling moment of hollow shell

$\mathrm{P}_{\mathrm{C}} \quad$ Axial failure load of shell \& core the core

$\mathrm{P}_{\mathrm{H}} \quad$ Axial failure load of the hollow shell $\sigma_{\mathrm{f}}$ material

$\mathrm{t}$ Thickness of shell with core $\zeta$ buckling $t_{\text {eq }} \quad$ Thickness of equivalent hollow

$\lambda_{\text {cr }} \quad$ Value of $\lambda$ minimizing critical

$v \quad$ Poisson's ratio of shell

$v_{c} \quad$ Poisson's ratio of core

$\rho \quad$ Density of the shell

$\rho_{c} \quad$ Density of the core

$\rho_{\mathrm{s}} \quad$ Density of the solid comprising

Critical failure stress of

$\zeta \quad$ Degree of ovalization at local

\section{Introduction}

Throughout history man has been intrigued by natural phenomena. Inventors have drawn inspiration from Nature to achieve some of the most influential developments in history, ranging from the utilization of composites in structures to the innovation of the airplane. Over the past few decades, interest in applying nature's model to engineering design has rapidly increased. This has been manifest in the creation of the field of biomimetics, which seeks to develop a better understanding of natural organisms and apply this understanding to improve engineering designs.

Thin-walled, cylindrical structures are found extensively in both natural organisms and engineering components. In nature, thin-walled structures are often subjected to a combination of an axial compressive load and a bending moment. Because of their innately efficient design, they are highly susceptible to buckling failure, so natural structures have been optimized to resist buckling failure. Plant stems, animal quills, and bird feather rachis all have a thin-walled, shell supported by a honeycomb- or foam-like cellular core, which increases their resistance to buckling (Fig. 1). A cellular core acts to reduce the weight of the structure without compromising the structural stability or load capacity. We examine the applicability of extending nature's model into engineering structures where minimizing the weight of the structure is an essential component of design, such as space shuttle fuel tanks, aircraft fuselages, and offshore oil platforms.

Previous studies of elastic buckling have suggested a thin-walled shell, supported by a compliant core, can achieve a higher buckling load than an equivalent hollow shell of the same weight and radius both for axial compression and pure bending. Karam and Gibson [2] analyzed the elastic buckling of a thinwalled, isotropic, cylindrical shell with a compliant elastic core to develop a 
simplified analysis for axisymmetric buckling in uniaxial compression and local buckling in pure bending. They determined the addition of a compliant core significantly increases the resistance of a shell to buckling failure.

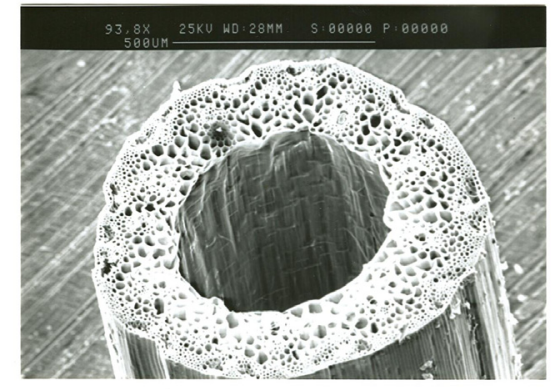

(a)

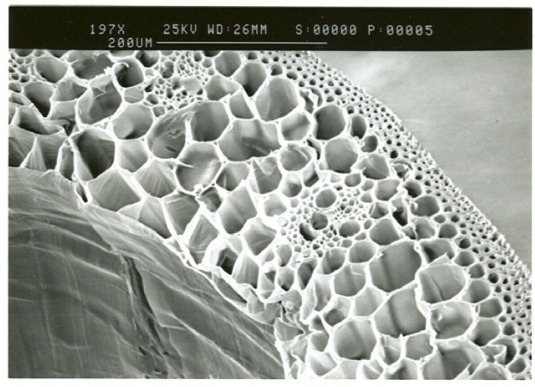

(b)

Figure 1: Micrographs of natural shell structure with compliant cores; Cross section of grass stem (Elytrigia repens) with a foam-like core.

In this paper, we extend the linear-elastic buckling theory from Karam and Gibson [2] by coupling basic plasticity theory to provide a more comprehensive analysis of isotropic, cylindrical shells with compliant cores. The goal is to examine the minimum weight design of thin-walled, cylindrical shells with compliant cores subjected to uniaxial compression and pure bending. For a given radius and length of the cylinder, required critical load, and shell and core materials, design equations for the shell thickness, the core thickness, and the core density that minimize the weight of the structure are presented. The improvement in the ratio of the critical load of the optimized shell with compliant core over an equivalent hollow shell of the same weight and radius is also examined for both uniaxial compression and pure bending, defined as $\mathrm{P}_{[\mathrm{C}]} / \mathrm{P}_{[\mathrm{H}]}$ and $\mathrm{M}_{[\mathrm{C}]} / \mathrm{M}_{[\mathrm{H}]}$, respectively. The optimized design for a shell with a compliant core presented here shows significant theoretical improvements over an equivalent hollow shell.

The design of natural, thin-walled structures with cellular cores is investigated and compared to this analysis. The functional utility, environmental stresses and material composition of natural structures is explored and contrasted with engineering designs.

This analysis further examines the feasibility of implementing compliant cores in thin-walled engineering structures where the weight to load bearing ratio is a critical element of design. The material and structural design constraints for this design in engineering structures are discussed, and the most advantageous engineering materials are presented. Based on the constraints, recommendations are developed and the potential for implementing compliant cores into thinwalled, engineering structures is discussed. 


\section{Optimal configuration}

The optimization analysis describes the minimum weight design of a thin-walled cylindrical shell with compliant, cellular-solid core loaded in uniaxial compression or pure bending. The analysis assumes the radius and length of the cylinder, the required load capacity, and the materials of the shell and the core are given. The values of the shell thickness, the core thickness, and the core density that minimize the weight of the structure are determined.

A thin-walled shell with a compliant core has an overall radius a, length $\mathrm{L}$, outer shell thickness $t$, inner core thickness $t_{c}$, and weight $w$. It is compared with an equivalent hollow cylinder of radius a, length $\mathrm{L}$, wall thickness $t_{\mathrm{eq}}$, and identical weight $w$ in (Fig. 2). The outer shell of the cylinder with the compliant core and the hollow cylinder are made of the same isotropic material, with density $\rho$, Young's modulus E, material failure strength $\sigma_{\mathrm{f}}$, and Poisson's ratio $v$. Similarly, the core has density $\rho_{\mathrm{c}}$, Young's modulus $\mathrm{E}_{\mathrm{c}}$, and Poisson's ratio $v_{\mathrm{c}}$.

\subsection{Assumptions}

We limit our analysis to thin-walled shells with large radius to thickness ratios, $\mathrm{a} / \mathrm{t}$. The materials under consideration are considered to behave linearly elastically up to the material failure, which we take to be deviation from linear elasticity. For simplification, Poisson's ratio has been evaluated for all of the figures and tables using $v=v_{c}=0.3$.

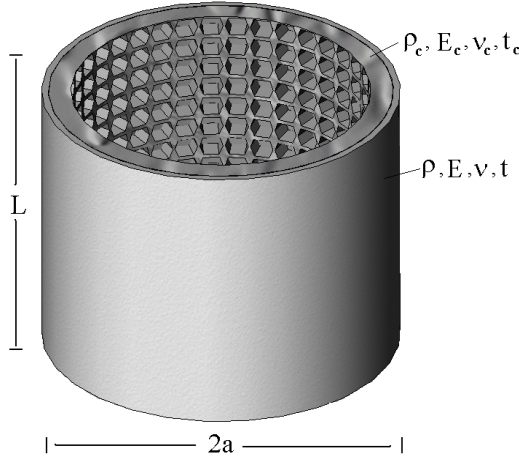

(a)

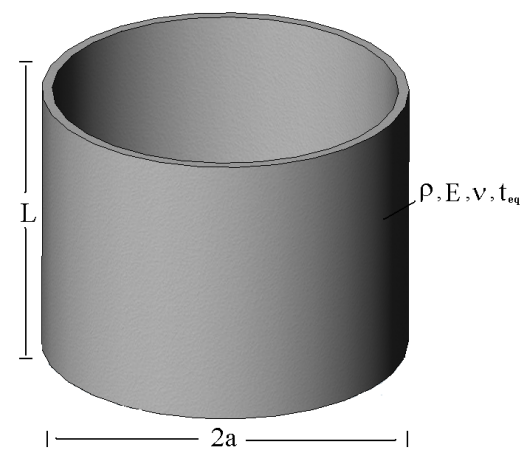

(b)

Figure 2: (a) A thin-walled cylindrical shell with a honeycomb core. (b) An equivalent thin-walled hollow cylindrical shell.

We also note that for the shell with a compliant core, the load is assumed to be entirely supported by the shell, and the compliant core behaves like an elastic foundation. This is justified by the fact that the core modulus in the plane of the load is negligible for both honeycomb and foam cores. According to Karam and Gibson [2], the stresses within the compliant core decay radially such that they become negligible at a depth into the core of 1.6 times the buckling half wavelength or $5 \lambda_{\text {cr. }}$. The thickness of the compliant core $t_{c}$ is taken to be this 
depth. The length of the shell $\mathrm{L}$ is also assumed to be at least several times the buckling half wavelength.

\subsection{Transition regions}

This analysis incorporates the possibility of material failure. If there is material failure of the hollow cylinder, then the axial load-carrying capacity of the cylinder with the compliant core will always be less than that of the hollow cylinder, since the shell thickness for the cylinder with the compliant core is always less than that of the corresponding hollow cylinder. A similar argument can be made for the case of pure bending where only negligible improvement is possible. Therefore, we only examine the two remaining failure scenarios where the hollow cylinder fails by elastic buckling and the corresponding shell with the compliant core fails by either material failure or elastic buckling. For the hollow shell to fail by elastic buckling eqn. (1) must be satisfied.

$$
\begin{array}{ll}
\frac{a}{t_{e q}} \geq \frac{E}{\sqrt{3\left(1-v^{2}\right)} \sigma_{f}} & \text { Uniaxial Compression } \\
\frac{a}{t_{e q}} \geq \frac{.939 E}{\pi \sqrt{1-v^{2}} \sigma_{f}} . & \text { Pure Bending }
\end{array}
$$

At lower ratios of $\mathrm{a} / \mathrm{t}_{\mathrm{eq}}$ material failure occurs in the hollow shell, while at higher ratios failure is by elastic buckling. Evaluating Poisson's ratio as 0.3 in eqn. (1b), shows the a/t transition for a hollow shell in pure bending is approximately one-half the value for axial compression. This lower transition allows for a larger variety of engineering materials to be used in the design of shells with compliant cores against failure in pure bending.

The transition of a shell with a compliant core, subjected to axial compression, between the buckling and material failure modes is found to depend only on the material properties of the shell and the core. Represented as a function of the core modulus to shell modulus ratio, it is given to be

$$
\left(\frac{E_{c}}{E}\right)_{A-\text { transition }}=\frac{2\left(1+v_{c}\right)\left(3-v_{c}\right)\left(\sqrt{1-v^{2}}\right)}{3}\left(\frac{\sigma_{f}}{E}\right)^{3 / 2} .
$$

As the ratio of the stiffness of the core to the stiffness of the shell is increased, the shell with the compliant core transitions to the material failure region. Assuming thin-walled structures with large a/t ratios, the transition for a shell with a compliant core, subjected to pure bending, between elastic buckling and material failure modes is given by

$$
\left(\frac{E_{c}}{E}\right)_{B-\text { transition }}=\left(\frac{2}{3}\right)\left(1+v_{c}\right)\left(3-v_{c}\right)\left(\sqrt{1-v^{2}}\right)\left(\frac{\sigma_{f}}{\left(1-\frac{3}{2} \zeta\right) E}\right)^{3 / 2} .
$$

For large $\mathrm{a} / \mathrm{t}$ ratios, the ovalization approaches zero, and the modulus transition ratios $\mathrm{E}_{\mathrm{c}} / \mathrm{E}_{\text {transition }}$ for both axial compression and pure bending are identical. 


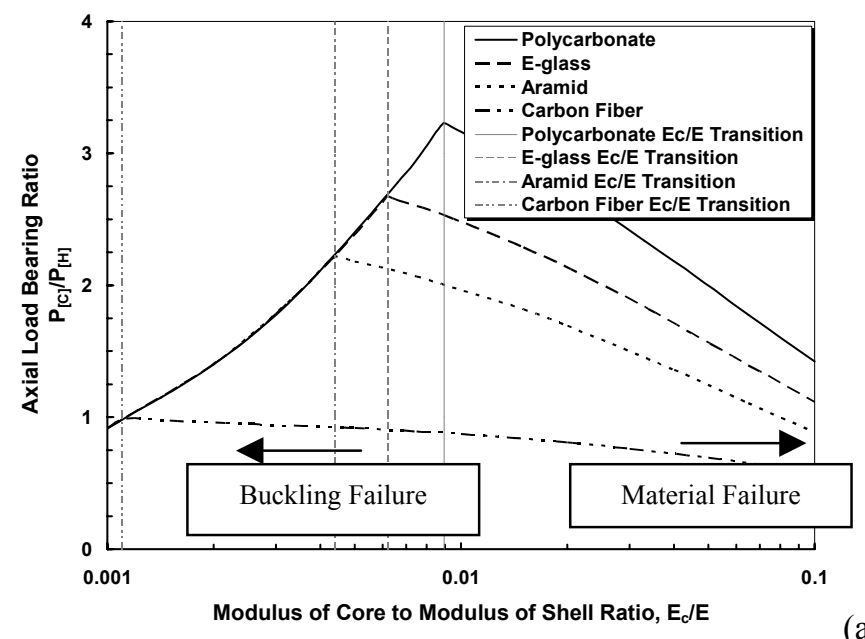

(a)

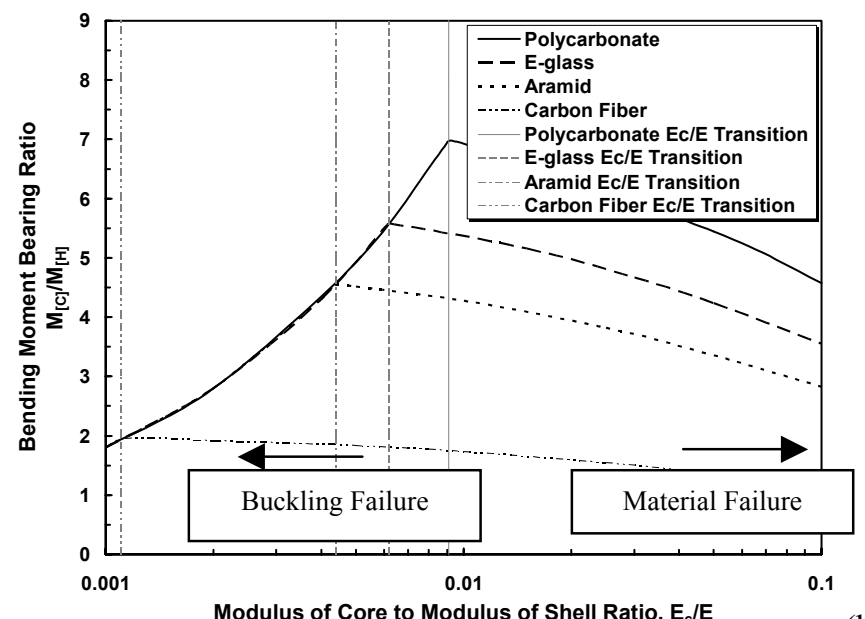

(b)

Figure 3: Load ratio plotted against the modulus ratio for a range of materials. For consistency, the shell and the honeycomb core are assumed to be made of the same material. All cylinders have equivalent radius to thickness ratios, $a / t=100$. (a) Uniaxial compression; (b) Pure bending.

\subsection{Design configurations}

The design problem presented contains non-linear equations with inequality constraints, requiring an analytical optimization for the minimum weight design to be a function of only one variable, which we selected to be the modulus ratio $\mathrm{E}_{\mathrm{c}} / \mathrm{E}$. Assuming large radius to thickness ratios, maximizing the axial load ratio, $\mathrm{P}_{[\mathrm{C}]} / \mathrm{P}_{[\mathrm{H}]}$ and bending moment ratio, $\mathrm{M}_{[\mathrm{C}]} / \mathrm{M}_{[\mathrm{H}]}$ in both the material failure region and the buckling failure region reveals the optimal value of the modulus ratio is 
always given by the transition modulus ratios in eqn. (2) and eqn. (3). The analysis assumes the shell and the core are made of the same material, but a parametric study reveals this analysis is valid for all engineering materials.

For large radius to thickness ratios, the optimal value for $E_{c} / E$, which maximizes the axial load ratio and moment ratio for both the material and buckling failure modes is given by

$$
\left(\frac{E_{c}}{E}\right)_{\text {optimum }}=\frac{2\left(1+v_{c}\right)\left(3-v_{c}\right) \sqrt{1-v^{2}}}{3}\left(\frac{\sigma_{f}}{E}\right)^{3 / 2} .
$$

This analysis demonstrates that optimization of the improvement in the load ratio and moment ratio is independent of whether or not the core is a honeycomb structure or a foam structure. The improvement for a shell with a honeycomb core over an equivalent hollow shell is significantly greater than that for a shell with a foam core for any given a/t ratio and material combination; therefore, the focus of this analysis is on shells with honeycomb cores. The optimal modulus ratio is also independent of the radius to thickness ratio, a/t because it is incorporated into the analysis through the constraint given by eqn. (1). Furthermore, the optimization is valid for all isotropic structural material combinations. Fig. 3 demonstrates the material independence with four common engineering materials and their corresponding $\left(\mathrm{E}_{\mathrm{c}} / \mathrm{E}\right)_{\text {transition values. }}$

Table 1 provided for the convenience of the reader, shows the material properties used for this analysis. The optimization based on the maximum improvement for a given design implies minimization of the weight for a prescribed load.

Table 1: $\quad$ Material properties.

\begin{tabular}{|c|c|c|c|}
\hline $\begin{array}{c}\text { Engineering } \\
\text { Material }\end{array}$ & Modulus, E (GPa) & $\begin{array}{c}\text { Material Failure } \\
\text { Stress, } \boldsymbol{\sigma}_{\mathbf{f}}(\mathbf{M P a})\end{array}$ & Density, $\mathbf{\rho}(\mathbf{g} / \mathbf{c m} \mathbf{3})$ \\
\hline Polycarbonate & 2.6 & 66 & 1.2 \\
\hline E-glass & 38 & 750 & 1.8 \\
\hline Aramid & 83 & 1300 & 1.4 \\
\hline Carbon Fiber & 220 & 1400 & 1.7 \\
\hline Titanium & 110 & 825 & 4.5 \\
\hline Aluminum & 69 & 240 & 2.7 \\
\hline
\end{tabular}

Sources: Data supplied by manufacturers and Shackelford [4].

Even though a shell with a compliant core may be optimally designed, it does not guarantee improvement over an equivalent hollow shell. A constraint for the minimum modulus ratio which results in improvement can be found. Assuming Poisson's ratios are given as $v=v_{c}=0.3$ and $t_{e q}$ is $15 \%$ larger than $t$ (from typical experimental data), a conservative guideline is given to be:

$$
\frac{E_{c}}{E}>\left[\frac{4}{3}\left(\frac{t}{a}\right)\right]^{3 / 2}
$$




$$
\frac{E_{c}}{E}>\left[\frac{2}{3}\left(\frac{t}{a}\right)\right]^{3 / 2} \cdot \quad \text { Pure Bending }
$$

For the cylinder with a compliant core to outperform an equivalent hollow cylinder the optimal modulus ratio given in eqn. (4) must satisfy eqn. (5). This equation is valid in both the buckling region and the material failure region. The minimum modulus ratio in pure bending is lower than the minimum modulus ratio in axial compression. This result is expected and supports the theory that a compliant core acts more to resist local buckling failure when subjected to pure bending than when subjected to axial compression. Therefore, we develop the optimal design of a cylinder with a compliant core against failure under axial compression.

Given that the optimal modulus ratio occurs when elastic buckling and material failure occur simultaneously, the optimal design for a shell with a compliant core can be determined. Equations (6-7) then give the core density, the shell thickness, and the core thickness that minimize the weight of a cylinder with compliant honeycomb or foam core for a prescribed axial load.

$$
\begin{aligned}
& \rho_{c}=\rho_{s} \frac{2\left(1+v_{c}\right)\left(3-v_{c}\right) \sqrt{1-v^{2}}}{3}\left(\frac{\sigma_{f}}{E_{s}}\right)\left(\frac{\sigma_{f}}{E}\right)^{1 / 2} \text { Honeycomb } \\
& \rho_{c}=\rho_{s} \sqrt{\frac{2\left(1+v_{c}\right)\left(3-v_{c}\right) \sqrt{1-v^{2}}}{3}}\left(\frac{\sigma_{f}}{E_{s}}\right)^{1 / 2}\left(\frac{\sigma_{f}}{E}\right)^{1 / 4} \quad \text { Foam } \\
& t=\frac{P}{2 \pi a \sigma_{f}} \quad t_{c}=\frac{5 P}{4 \pi a \sigma_{f}}\left[\frac{E}{\left(1-v^{2}\right) \sigma_{f}}\right]^{1 / 2}
\end{aligned}
$$

\section{Nature's design}

A study of thin-walled, natural structures with a cellular-solid core was conducted by Karam and Gibson (1994b). We examine the animal quills and spines, which most closely match the shell with compliant core designs discussed in this analysis.

\subsection{Natural design vs. optimal configuration}

The primary constituent of animal quills and spines is a family of proteins called keratin. While various types of keratin can co-exist in the same structure, quills and spines are taken to be composed of $\alpha$-keratin. The mean failure stress and elastic modulus of compact alpha-keratin is taken to be $226 \mathrm{MPa}$ and $2.52 \mathrm{GPa}$, respectively (Crenshaw, 1980). Based on these values, the optimal modulus ratio and the minimum modulus ratio in axial compression and bending can be determined. Table 2 demonstrates a comparison of the optimal modulus ratio to 
the actual modulus ratio found experimentally (after Karam and Gibson [3]). The average experimental modulus ratio is 0.061 with a standard deviation of 0.036. The optimal modulus ratio of 0.060 demonstrates natural structures closely approach the analytical optimal design presented in this analysis. The apparently large standard deviation about the mean is expected. Natural structures are designed differently than engineering structures. In nature, porous structures are found to have extremely small cell-sizes on the order of 10-100 microns, which may be necessary for structural support during formation. Engineering structures are, practically, manufactured with much larger cell-sizes. In addition, natural structures often serve to provide greater utility than support alone supplying nutrients, providing the thermal regulation, and allowing for communication. Accounting for all of these functions surely will cause deviation from the structural optimization. Moreover, the material properties of keratin are highly dependent on temperature, humidity, and mechanical conditions, and keratin itself is a complex structure. Keratin is often modeled as a multiphase structure with a large range of elastic modulus; nearly all of the experimental data is within the range of corresponding optimal modulus ratios. Therefore, the functional utility of each structure, the environmental stresses acting on each structure, and the composition of the keratin in each species may account for the differences in core configuration, variations in the modulus ratio, and large standard deviation in the modulus ratio.

Table 2: Comparison of experimental, optimal, and minimum modulus. ratios for animals.

\begin{tabular}{|c|c|c|c|c|c|c|}
\hline $\begin{array}{c}\text { Animal } \\
\text { (genus/species) }\end{array}$ & $\begin{array}{c}\text { Common } \\
\text { Name }\end{array}$ & $\begin{array}{l}(\mathrm{a} / \mathrm{t}) \\
\text { ratio }\end{array}$ & $\begin{array}{c}\text { Experim. } \\
\left(\mathbf{E}_{\mathrm{c}} / \mathbf{E}\right) \\
\end{array}$ & $\begin{array}{c}\text { Optima } \\
1 \\
\left(E_{c} / E\right)_{O p} \\
t \\
\end{array}$ & $\begin{array}{c}\text { Axial } \\
\left(\mathbf{E}_{\mathrm{c}} / \mathbf{E}\right)_{\mathrm{Mi}} \\
\mathrm{n}\end{array}$ & $\begin{array}{c}\text { Bendin } \\
g \\
\left(\mathbf{E}_{\mathrm{c}} / \mathbf{E}\right)_{\mathrm{Mi}} \\
\mathrm{n}\end{array}$ \\
\hline Coendou Prehensilis & Braz. Porc. & 14.0 & 0.040 & 0.060 & 0.029 & 0.010 \\
\hline Erethizon & NA Porc. & 18.0 & 0.016 & 0.060 & 0.020 & 0.007 \\
\hline $\begin{array}{c}\text { Tachyglossus } \\
\text { Aculeatus } \\
\end{array}$ & Echidna & 2.3 & 0.012 & 0.060 & 0.440 & 0.156 \\
\hline Hystrix Galeata & Echidna & 17.6 & 0.047 & 0.060 & 0.022 & 0.008 \\
\hline Hystrix Idica-Cristata & Porcupine & 9.5 & 0.052 & 0.060 & 0.052 & 0.018 \\
\hline Hystrix Subristatus & Porcupine & 10.0 & 0.081 & 0.060 & 0.048 & 0.017 \\
\hline Erinaceus Europaeus & Hedgehog & 13.7 & 0.100 & 0.060 & 0.030 & 0.011 \\
\hline Erinaceus Europaeus & Hedgehog & 12.5 & 0.100 & 0.060 & 0.035 & 0.012 \\
\hline Hemichinus Spinosus & Spiny Rat & 12.7 & 0.100 & 0.060 & 0.033 & 0.012 \\
\hline
\end{tabular}

*After Karam and Gibson [3]. 


\section{Material design}

The feasibility of this design has been examined for a wide variety of materials. The constraint given by eqn. (1) indicates materials with low $E / \sigma_{\mathrm{f}}$ ratios are ideal for the shell of the cylinder with the compliant core, such as polymers or select composites. Table 3 further demonstrates the importance of the ratio of the elastic modulus to the material failure strength in the shell material, referred to here as the intrinsic stability ratio. Shells made of materials with a low intrinsic stability ratio have the most substantial improvement in the axial load ratio and bending moment ratio from the addition of a compliant core.

Table 3: Load ratio and moment ratio for variable intrinsic stability ratios.

\begin{tabular}{|c|c|c|c|}
\hline Engineering Material & $(\mathbf{E} / \sigma \mathbf{\sigma}) /\left(\mathbf{E} / \sigma_{\mathbf{v}}\right)_{[\mathbf{P o l y c a r b o n a t e}}$ & $\mathbf{P}_{\left[\mathbf{C} / \mathbf{P}_{[\mathbf{H}]}(\mathbf{a} / \mathbf{t}=\mathbf{1 0 0})\right.}$ & $\mathbf{M}_{[\mathbf{C}]} / \mathbf{M}_{[\mathbf{H ]}}(\mathbf{a} / \mathbf{t}=\mathbf{1 0 0})$ \\
\hline Polycarbonate & 1.0 & 3.2 & 7.0 \\
\hline E-glass composite & 1.3 & 2.7 & 5.6 \\
\hline Aramid composite & 1.6 & 2.1 & 4.5 \\
\hline Titanium & 3.4 & 1.2 & 2.3 \\
\hline Carbon Fiber composite & 4.0 & 1.0 & 2.0 \\
\hline Aluminum & 7.3 & 1.0 & 1.0 \\
\hline
\end{tabular}

The stability ratios, $\mathrm{E} / \sigma_{\mathrm{y}}$ are normalized by that of polycarbonate. The shell and the core are assumed to be made of the same material.

\section{Discussion}

Analyzing natural phenomena has provided insight into engineering design throughout the millennia. This analysis discusses the applicability of extending nature's model of thin-walled shells supported by a compliant, cellular core into engineering structures. The optimization of a shell with a compliant core displays promising results. We discovered the optimal configuration occurs when the shell with a compliant core is designed to fail in elastic buckling and material failure simultaneously. Tractable equations, which can be used to optimize the design of a cylinder with a compliant core based on a minimum weight design are provided. The constraints on the optimal modulus ratio reveal materials with the most potential for practical implementation have relatively low intrinsic stability ratios, such as polymers or select composites.

Natural, thin-walled structures provide support for much of the analysis. Furthermore, hedgehog spines, which exhibit a core structure resembling the honeycomb design, prove to have a greater resistance to buckling than porcupine quills, which exhibit a core structure resembling the foam design. Experiments have also shown that the average modulus ratio of animal quills and spines is nearly identical to the optimal modulus ratio discussed here and may only differ due to the need for functionality in addition to structural support. Moreover, natural structures also reveal shells with compliant cores are more effective at 
increasing the resistance to buckling when subjected to a bending moment than when subjected to an axial load.

\section{Conclusion}

Theoretically, the optimized shell with a compliant core demonstrates substantial improvement in weight savings over a comparable hollow shell. However, this design is only practical for implementation in structures requiring large radius to thickness ratios, where the weight to load ratio is a critical element of design. Therefore, it is feasible to consider implementation of this design in relatively large scale engineering structures, such as space shuttle fuel tanks, aircraft fuselages, and offshore oil platforms. Natural structures support this optimization, indicating the optimized shells with compliant cores have enormous potential to be a competitive technology in select engineering structures.

\section{Acknowledgements}

Financial support for this project was provided by the National Science Foundation (Grant Number CMS-0408259), for which we are grateful. This research was performed while on appointment as a U.S. Department of Homeland Security (DHS) Fellow under the DHS Scholarship and Fellowship Program, a program administered by the Oak Ridge Institute for Science and Education (ORISE) for DHS through an interagency agreement with the U.S Department of Energy (DOE). ORISE is managed by Oak Ridge Associated Universities under DOE contract number DE-AC05-00OR22750. All opinions expressed in this paper are the author's and do not necessarily reflect the policies and views of DHS, DOE, or ORISE.

\section{References}

[1] Crenshaw, D.G. (1980). The Mechanical Properties of Biological Materials. Symposia Soc. Exp. Biology XXXIV. Cambridge University Press, Cambridge.

[2] Karam, G. N. and Gibson, L. J. (1994). Elastic buckling of cylindrical shells with elastic cores-I. analysis. Int. J. Solids Structures 32, 1259-1283.

[3] Karam, G. N. and Gibson, L. J. (1994). Biomimicking of animal quills and plant stems: natural cylindrical shells with foam cores. Material Sci. \& Eng. C2, 113-132.

[4] Shackelford, J. (2000). Material Science for Engineers. Prentice Hall, New Jersey. 